\title{
Vertical structure of stellar populations in galaxy disks
}

\author{
David Streich, Roelof S. de Jong and the GHOSTS team \\ Leibniz-Institut für Astrophysik Potsdam (AIP), An der Sternwarte 16, 14486 Potsdam, \\ Germany \\ email: dstreich@aip.de, rdejong@aip.de
}

\begin{abstract}
Stellar populations are most useful for disentangling formation and evolution histories of galaxies. We present here results obtained using data from the GHOSTS survey (RadburnSmith et al., 2011) which uses HST photometry to resolve stellar populations in nearby massive disk galaxies. Using color magnitude diagrams we can distingiush stellar populations of different ages and analyse the spatial structure of each population seperately.

We have examined the vertical disk structure in six edge-on galaxies. We find a general heating of disk, i.e. larger scaleheights for older populations. The scaleheight of each population is constant over most of radial extent of each galaxy.

In massive galaxies $\left(V_{\text {rot }}>150 \mathrm{~km} / \mathrm{s}\right)$ we clearly see a thick component (i.e. there are more stars at large distances from the plane than expected from a single disk model). These thick components consist of intermediate-aged and old stars ( $>1$ Gyr), and the (thick) scaleheight of the old population (>4 Gyr) is significantly larger than the (thick) scaleheight of the intermediate aged (1-2 Gyr) population.

This finding argues against a rapid formation of the thick components and favors a more secular formation of these components.
\end{abstract}

Keywords. galaxies: spiral, galaxies: stellar content, galaxies: structure, galaxies: evolution

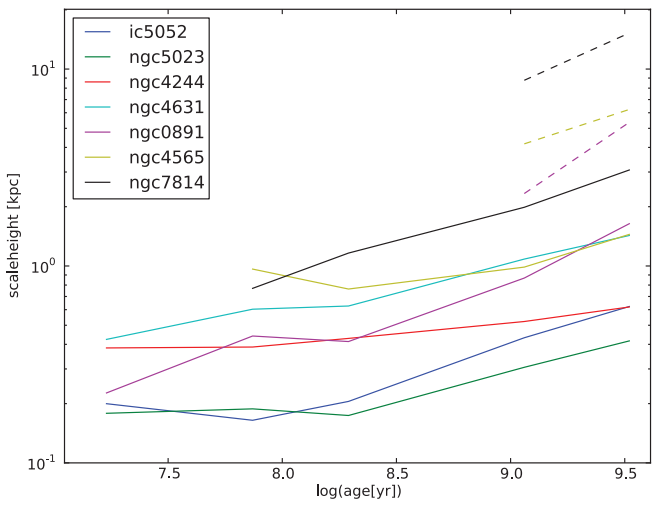

Figure 1. Scaleheights as a function of age. solid line: (thin) disk, dashed line: thick component. A clear increase with age is seen for both the thin and the thick components. ( $A$ color version of this figure is available in the online journal.)

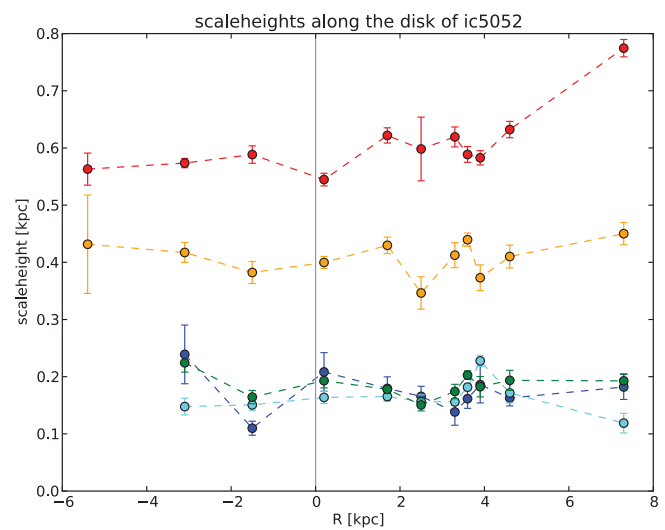

Figure 2. Scaleheights as a function of radius in IC 5052. Different lines show different population. With the exception of the outermost point if the old populations, the scaleheights are constant. (A color version of this figure is available in the online journal.)

\section{Reference}

Radburn-Smith, D. J., et al. 2011, ApJS 195, 18 